\title{
Effects of a Static Magnetic Field on Mineralization of MC3T3-E1 Cells
}

\author{
Yoko Imaizumi, DDS, ${ }^{a}$ Shogo Ozawa, DDS, PhD, ${ }^{a}$ Koji Hirukawa, DDS, PhD, ${ }^{b}$ \\ Akifumi Togari, PhD, ${ }^{b}$ and Yoshinobu Tanaka, DDS, $\mathrm{PhD}^{\mathrm{a}}$ \\ ${ }^{a}$ The First Department of Prosthodontics, School of Dentistry, Aichi-Gakuin University, Nagoya, Japan \\ ${ }^{\mathrm{b}}$ Department of Pharmacology, School of Dentistry, Aichi-Gakuin University, Nagoya, Japan
}

\section{Clinical significance}

Static magnetic field (SMF) has been utilized for the retention of removable partial dentures. This study indicated that an SMF influences not only the mechanical attachment but also promotes osteoblast-like cells calicification. Although these effects of an SMF remain to be biologically understood, increased in vitro bonelike formation suggests its future applicability.

\begin{abstract}
Purpose: The stage at which bone formation was influenced by a static magnetic field (SMF) remains to be elucidated. The purpose of this study was to investigate the initial and long-term effects of moderateintensity SMF on mouse osteoblastic cells (MC3T3-E1). Methods: MC3T3-E1 osteoblastic cell cultures were exposed to SMF (250 mT, Nd-Fe-B disc magnet). The long-term effect of this exposure was evaluated by measuring mineralized nodule formation. Osteoblastic cell proliferation was assessed using a colorimetric proliferation assay (WST-8); differentiation was evaluated by measuring alkaline phosphatase (ALP) activity; and noncollagenous gene expression was evaluated using real-time PCR to determine the initial and early responses.
\end{abstract}

Results: After a month of continuous SMF exposure, mineralized nodule formation increased significantly. The initial proliferative activity decreased and was not related to apoptotic changes. ALP activity and the gene expression levels of secreted phosphoprotein 1 (SPP1), integrin binding sialoprotein, and bone gla protein were not influenced by the SMF exposure.

Conclusion: Moderate-intensity $(250 \mathrm{mT}) \mathrm{SMF}$ exposure increased mineralized nodule formation in mouse osteoblastic MC3T3-E1 cells. However, gene expression related to decreased cell proliferation and unaltered cell differentiation and bone matrix did not

Corresponding to: Dr Yoko Imaizumi

The First Department of Prosthodontics, School of Dentistry, Aichi-Gakuin University

2-11 Suemori-dori, Chikusa-ku, Nagoya 464-8651, Japan

Tel\&Fax: +81-52-759-2152

E-mail: youko@dpc.aichi-gakuin.ac.jp

Received on October 30, 2006 / Accepted on February 1, 2007 correlate with the long-term effects observed following SMF exposure. The mechanism by which SMF exposure influences osteoblast mineralization remains unknown.

Key words: static magnetic fields, osteoblast, mineralization, apoptosis

\section{Introduction}

Magnetic attachments for removable partial dentures (RPDs) were introduced 3 decades ago. ${ }^{1,2}$ Since the introduction of rare earth element magnets, the use of magnetic attachments has increased and has become one of the treatment modalities for designing an RPD for cases with multiple missing teeth. ${ }^{3}$ It has been clinically proven that a benefit of magnetic attachment involves an increase in retention and stability of an RPD; further, the decrease in harmful lateral forces facilitates the long-term survival of the abutment teeth. ${ }^{4}$ On the other hand, shortcomings of the magnetic attachments include a mandatory root canal treatment and interference for diagnostic tools that use magnetic resolution. Although the recently designed attachments employ closed magnetic circuits, magnetic flux leakage cannot be eliminated.

The biological effects of the static magnetic field (SMF) remain unclear and depend on various factors such as the intensity of the magnetic field, exposure conditions, and the types of organs, tissues, and cells being treated. With regard to bone tissue effects, several studies have reported the stimulation of bone formation and osteoblast proliferation and differentiation., ${ }^{5,6}$ However, other studies have reported regarding the suppressive effects of an SMF on osteoblasts. ${ }^{7}$ Therefore, there is still lack of understanding regarding the cell based mechanism of SMF exposure. 

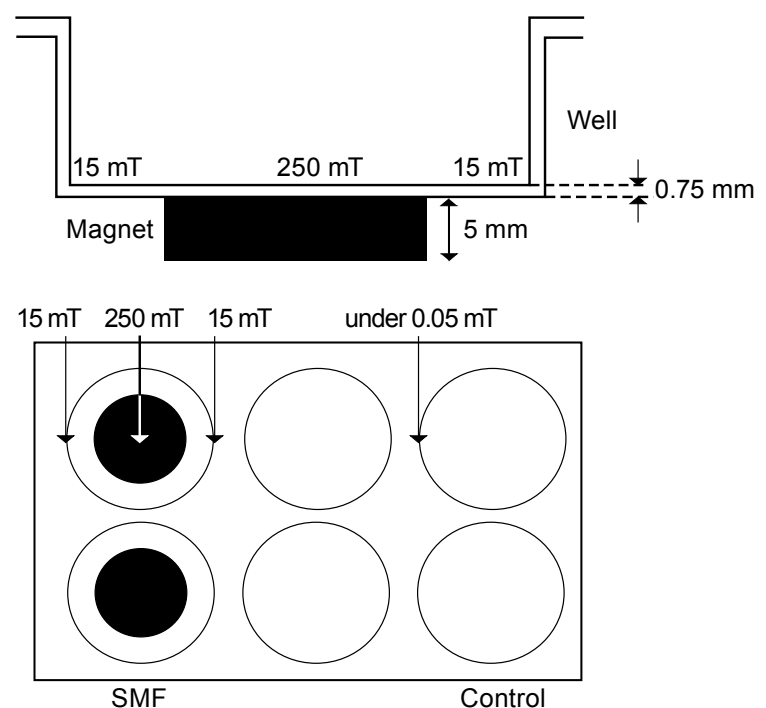

Fig. 1 Static magnetic field exposure system.

Bone formation is a sequential event involving osteoblasts and proceeds via 3 stages, namely, proliferation, differentiation, and matrix mineralization. Each stage involves genetic and transcriptional factors that strongly promote or inhibit bone formation. For instance, core-binding factor A1 (Cbfa1) is an essential factor in osteoblast differentiation that genetically regulates the osteoblast phenotype, ${ }^{8,9}$ and osteocalcin is a bone matrix protein that is released simultaneously along with the onset of mineralization. ${ }^{10,11}$ The stage at which bone formation is influenced by an SMF remains to be elucidated. The purpose of this study was to investigate the initial and long-term effects of a moderate-intensity SMF on mouse osteoblast-like cells (MC3T3-E1).

\section{Materials and methods}

\section{Static Magnetic Field Exposure System}

In the present study, neodymium-iron-boron magnet disks $(20.0 \mathrm{~mm}$ in diameter and $5 \mathrm{~mm}$ in thickness; NE086, 26 SEISAKUSYO Co., Ltd. Kyoto, Japan) and 6-well plastic culture plates were used. The cultures were exposed to the magnet by placing the magnet below the wells. The N-pole was directed toward the culture but did not come in contact with it. The magnetic flux density was monitored using a Tesla meter (TM-601, KANETEC Co., Ltd., Nagano, Japan) present at the bottom of each well, where the mouse osteoblastic cells (MC3T3-E1 cells) attached themselves to the culture plates. The magnetic field demonstrated a maximum flux strength of $250 \mathrm{mT}$. The cells in the SMF group were subjected to continuous SMF exposure. The culture plate of the control group and that of the experimental group were placed next to each other in the same incubator. The flux density values of the control culture plates were observed to be no greater than $0.05 \mathrm{mT}$, that is, it was the same level as that of the natural magnetic field on the earth (Fig. 1).

\section{Cell Culture}

The MC3T3-E1 cells, derived from newborn mouse calvaria, are a clonal osteogenic cell line whose cells can differentiate into osteoblasts. The cells were plated in the 6 -well plates at a concentration of $1 \times 10^{4}$ cells/well. These cells were cultured in plastic plates containing $\alpha$-minimal essential medium ( $\alpha$-MEM) supplemented with $10 \%$ fetal calf serum, $100 \mathrm{IU} / \mathrm{ml}$ penicillin, 100 $\mu \mathrm{g} / \mathrm{ml}$ streptomycin, $10 \mathrm{mM} \beta$-glycerophosphate, and $50 \mu \mathrm{g} / \mathrm{mL}$ of ascorbic acid at $37^{\circ} \mathrm{C}$ in a humidified atmosphere containing $5 \% \mathrm{CO}_{2}$ in air. SMF exposure was initiated when the culture reached $70 \%$ confluence. The medium in each plate was changed every 3 days.

\section{Bone Nodule Formation Assay}

With regard to the bone nodule formation assay, mineralized extracellular matrices were stained using the von Kossa technique. The cells were then washed 3 times with PBS, followed by fixation in ethanol for $10 \mathrm{~min}$. Subsequently, they were washed 3 times each with PBS and distilled water, and then stained with $5 \% \mathrm{AgNO}_{3}$ solution for $10 \mathrm{~min}$. After staining, the cells were washed 3 times with distilled water and fixed in a $3 \%$ $\mathrm{Na}_{2} \mathrm{~S}_{2} \mathrm{O}_{3}$ solution for $10 \mathrm{~min}$. The area and number of calcified nodules stained by the von Kossa technique were digitally photographed and measured using the NIH image software.

\section{Proliferation Assay}

Cell proliferation was assessed using a colorimetric proliferation assay (WST-8 Cell Counting Kit, Dojindo, Kumamoto, Japan). We determined the hormazan dye content in the samples by measuring the absorbance of the 1-, 4-, and 7-day cultures at $450 \mathrm{~nm}$. The measurements were performed after changing the medium by applying the hormazan solution for $30 \mathrm{~min}$ to $1 \mathrm{~h}$, depending on the change in color. 


\section{DNA Fragmentation Assay}

With regard to the cellular DNA fragmentation assay, solid-phase immobilized anti-DNA mAb and anti-BrdU mAb labeled with peroxidase were used to detect BrdU-labeled DNA fragments in the cytoplasm of the cell lysates (Cellular DNA Fragmentation ELISA, Roche Molecular Biochemicals, Mannheim, Germany). In brief, the MC3T3-E1 cells grown in culture dishes $\left(1 \times 10^{5}\right.$ cells/ml) were labeled with BrdU for $24 \mathrm{~h}$, the cell concentration was then adjusted to $5 \times 10^{4}$ cell $/ \mathrm{ml}$, and $100 \mu \mathrm{l}$ of the suspension was transferred to each well of a microtiter plate (96-well, flat bottom). TNF- $\alpha$-treated cells] were used as the positive control. The concentration of BrdUrd-labeled DNA released into the cytoplasm of the apoptotic cells was quantified by ELISA.

\section{Alkaline Phosphatase Activity and Protein Assays}

Alkaline phosphatase (ALP) activity was determined using the BluePhos Microwell Phosphatase Substrate System (Kirkegaard \& Perry Laboratory, Gaithersburg, USA). Briefly, the samples of the supernatants were added to the substrate solution and reacted for $30 \mathrm{~min}$. The products were detected at $595 \mathrm{~nm}$ by using a spectrophotometer. Protein concentration of the cell lysate was estimated using the Bradford method (BioRad Laboratories). The ALP activity was normalized to that of the total protein content.

\section{Real-Time Transcript Analysis}

Total RNA was extracted using Qiazol (QIAGEN, California, USA) ${ }^{12}$ and genomic DNA was eliminated by DNase I treatment. The quality and quantity of RNA was measured by spectrophotometry. Subsequently, an Oligo-dT primer and the Moloney murine leukemia virus reverse transcriptase (Gibco-BRL; Grand island NY, USA) were used in a reverse transcriptase reaction to obtain cDNA. The expression level of secreted phosphoprotein 1 (SPP1, osteopontin), integrin-binding sialoprotein (Ibsp), and bone gla protein (BGP, osteocalcin) mRNAs was determined by performing quantitative real-time PCR using a sequence detector (ABI 7700; Applied Biosystems, Foster City, USA). The primers and Taq Man probes were designed according to the UniGene IDs (Applied Biosystems, Foster City, USA); the primers for SPP1(OPN) and Ibsp were NM_009263, and NM_008318, respectively.

The reaction mixture containing the cDNA sample was prepared according to the manufacturer's protocols to yield a final concentration of $1 \times$ TaqMan Universal PCR MasterMix and 200 $\mathrm{nM}$ of each primer. The reaction mixture also contained target hybridization probes $(250 \mathrm{nM}$ each). These probes were labeled at the $5^{\prime}$ end with the reporter fluorescent dye 6-carboxyfluorescein (FAM). The thermal cycling conditions were as follows: $50^{\circ} \mathrm{C}$ for $2 \mathrm{~min}$ and $95^{\circ} \mathrm{C}$ for 10 min, followed by 40 cycles of amplification at 95 ${ }^{\circ} \mathrm{C}$ for $15 \mathrm{~s}$ and denaturing and annealing-extension at $60^{\circ} \mathrm{C}$ for $1 \mathrm{~min}$. All data were calculated by a standard curve, and the results were standardized by comparison with the endogenous control (TaqMan Rodent GAPDH Control Reagents kit, Applied Biosystems, Foster City, USA).

\section{Statistical Analysis}

Values are presented as means \pm S.E. with $4-6$ cul- $^{-}$ tures per group. Each experiment was repeated 3 times. Differences between the control and experimental groups were assessed by paired $\mathrm{Stu}^{-}$ dent $t$ test.

\section{Results}

\section{Analysis of Mineralization}

After maintaining the culture for 3 weeks, mineralized nodules appeared on the cell layers. The culture was stopped on Day 31 and the visualization of mineralized nodules became clearer by Von Kossa staining (Fig. 2). The experimental culture displayed scattered black stained nodules that were equally distributed in the culture dishes. On the other hand, the control culture displayed sparse nodule formation. The results of image analysis revealed that the total mineralized area had increased significantly $(P<0.05)$. Moreover, the average number of nodules had also increased significantly $(P<0.01)$.

\section{Proliferation and Apoptosis Assay}

The effect of SMF contentious exposure in the early phase was determined by the proliferation assay. The results were normalized using the corresponding control culture, and these results indicated the relative ratio against the control. With regard to proliferative activity, the 1-day culture did not reveal any difference, but the $4^{-}$ and 7-day cultures demonstrated a significant decrease $(P<0.05)$ of approximately 10\% (Fig. 3). The DNA fragmentation assay was performed in 


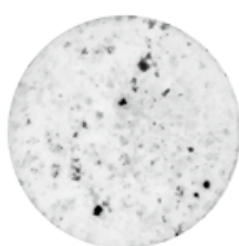

Control

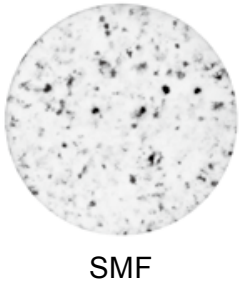

(a) von Kossa staining (31th days)

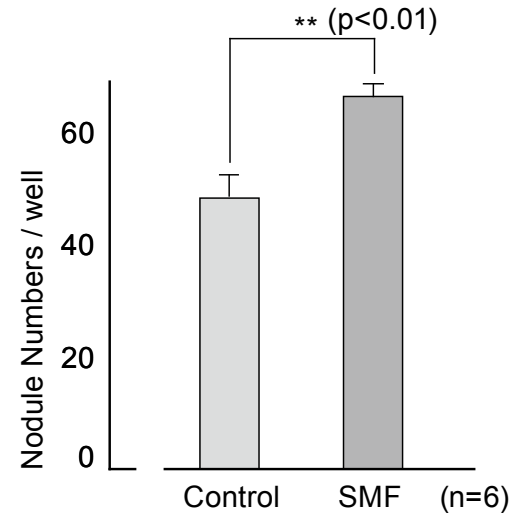

(b) Nodule numbers

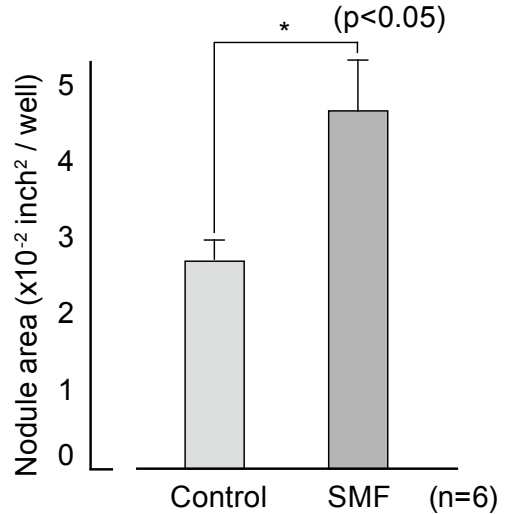

(c) Nodule area

Fig. 2 Mineralized nodule formation (a) von Kossa staining (day 31), (b) Number of nodules, (c) Area occupied by the nodules.

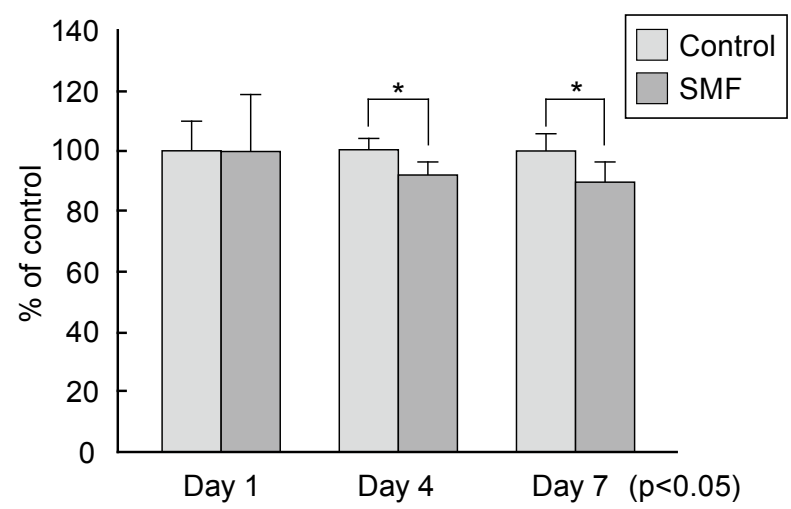

Fig. 3 Cell proliferation activity.

order to assess the relationship between partial inhibition of proliferation and apoptosis due to SMF exposure. Consequently, identical levels of fragmentation were observed in 1- and 3-day cultures and the control. Challenge with TNF- $\alpha$ represented the positive control for apoptosis (Fig. 4).

\section{Differentiation Assay}

Osteoblast differentiation is characterized by an increase in in vitro ALP activity. Indeed, MC3T3-E1 osteoblast-like cells gradually increase ALP activity until Day 4. However, SMF exposure had little effect on ALP activity (Fig. $5)$. Another marker of osteoblast differentiation is the expression of noncollagenous proteins, including SPP1, Ibsp, and BGP. After $24 \mathrm{~h}$ of SMF exposure, the mRNA expression levels were quantified using real-time PCR. These results are illustrated in Figure 6. However, SMF exposure did not alter any of the expression levels.

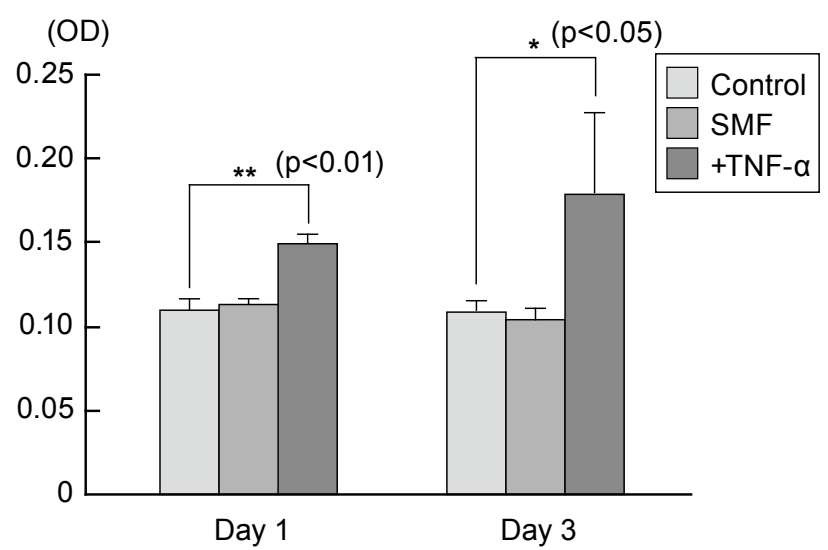

Fig. 4 DNA fragmentation assay (apoptosis).

\section{Discussion}

Although the effects of SMF have been studied in various tissue cultures, the results of previous studies are conflicting. The growth of HeLa cells displayed no difference after $120 \mathrm{mT}$ SMF exposure. ${ }^{13}$ On the other hand, Feinendegen and Muhlensiepen reported that a 1.4-T exposure for 30 min promoted the tyrosine kinase activity in mouse bone marrow cells. Contrary to the above observation, Butler and Dean demonstrated that 0.4 T SMF exposure had inhibitory effects on KB cells. ${ }^{15}$ Therefore, it is suggested that the effect of SMF depends on the duration of SMF exposure, tissue penetration, and heat generation. ${ }^{14-16} \mathrm{Us}^{-}$ ing mouse osteoblastic cells, this study has focused on the long-term effect of continuous SMF exposure and the early responses to SMF.

In this study, we used rare earth magnets, including Neodym (Nd), Iron (Fe) and Boron (B) that are similar to those used in dental magnetic attachments. The magnetic fluxes of the culture wells were unevenly distributed. SMF strength 


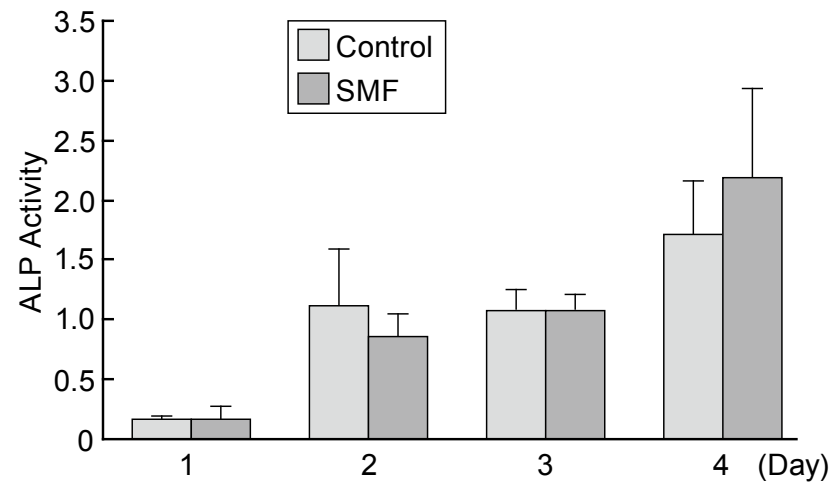

Fig. 5 Measurement of ALP activity.

was the highest at the center of the magnet (approximately $250 \mathrm{mT}$ ) and the lowest at rim of the well (approximately $15 \mathrm{mT}$ ). Clinically, the maximum strength of the attachment surface is 100 $\mathrm{mT}$. The SMF exposure simulates periodontal tissue reaction, particularly with regard to bone formation, when the magnet is placed close to the bone.

Regarding the effect of an SMF on the osteoblasts, cell growth and differentiation were the focus because the therapeutic benefits of SMF exposure have been confirmed by clinical results in orthopedics. Hence, the mechanism of the magnetic effect was studied in vitro to elucidate its effect. Indeed, osteoblasts, which are responsible for bone formation, are sensitive to SMF exposure. However, the effects of SMF exposure were inconsistent. In well-differentiated osteoblastic cells (ROS17/2.8, UMR106, rat calvaria cells), $160 \mathrm{mT}$ SMF exposure did not increase cell growth; instead, it promoted ALP activity and mineralization. ${ }^{6}$ Camillreri and Macdonald reported that $100 \mathrm{mT}$ SMF exposure decreased the proliferation of the rat calvaria osteoblastic cells. ${ }^{7}$ In this study, we observed that $250 \mathrm{mT}$ SMF exposure decreased cell proliferation and did not increase ALP activity in the mouse osteoblastic MC3T3-E1 cells. Since this cell line possesses pre-osteoblastic cell characteristics, the effect of SMF exposure is dependent on the stage of osteoblastic differentiation.

It has been suggested that SMF causes morphological alterations in cell shape ${ }^{17}$ and bioelectrical properties, including the influence of transmembrane calcium flux. ${ }^{18}$ An increase in $\left[\mathrm{Ca}^{2+}\right]$ influx might trigger the development of apoptosis. In this study, we observed that SMF partially inhibited cell growth in cell cultures in which apoptotic cells were not detected. Teodori

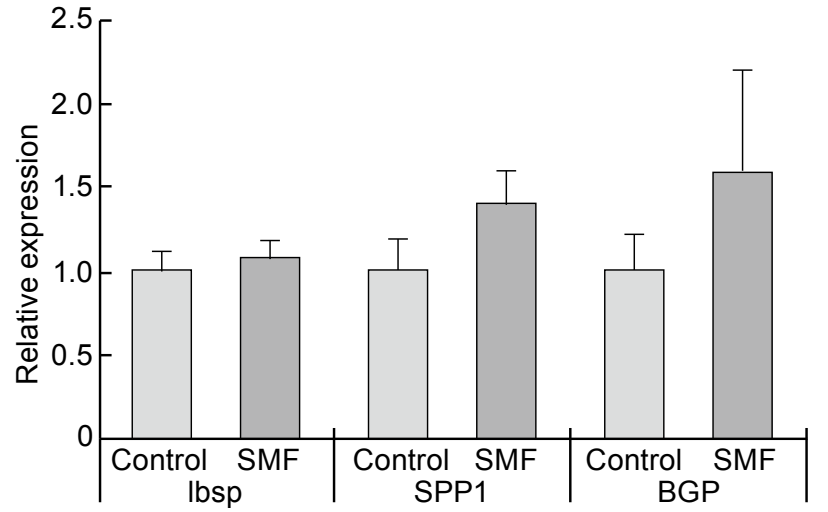

Fig. 6 mRNA expression after $24 \mathrm{~h}$ of SMF exposure.

reported that $6 \mathrm{mT}$ SMF exposure modulated $\mathrm{Ca}^{2+}$ fluxes in human glioblastoma cells and interfered with the stress-induced apoptotic pathway. ${ }^{19}$ Therefore, our results were consistent with those of a previous report that demonstrated that SMF alone did not exert apoptogenic activity.

The final outcome observed in the osteoblastic culture was mineralization, which involves the proliferation and differentiation stages, and is essential for mineralization of the extracellular matrix and inorganic phosphate. During the stages in bone formation, the expression of noncollagenous proteins such as SPP-1, Ibsp, and bone glaprotein plays an important role in calcified matrix formation. We also observed that SMF increased mineralized nodule formation; however, the expression of the genes of noncollagenous proteins involved in bone formation were not elevated, and their expression levels did not have an influence on alkaline phosphatase activity, which is believed to be involved in the extracellular breakdown of pyrophosphate, a potent inhibitor of calcium and phosphate deposition. ${ }^{20}$ Therefore, it is suggested that the SMF-induced mineralization did not mediate cellular gene alteration. Since these results are not in agreement with those of a previous study of a similar nature, ${ }^{6}$ it is possible that factors other than cell proliferation and differentiation, such as apoptosis and matrix-related gene expression, did not induce this long-term effect following SMF exposure. Further study is required to clarify the biological and physiological mechanism of SMF exposure.

Clinical application of SMF exposure is limited to diagnostic appliances, such as MRI, and its physiological effects include relief from pain or controlling of blood pressure. ${ }^{21}$ Since 1992 , magnetic attachments used for oral treatments have found greater application. However, their design 
did not permit the tissue around the attachment to be exposed to SMF; our results suggest that magnetic leakage might not be a risk. Further, with regard to the alveolar bone, it would be favorable to stimulate bone formation by SMF exposure using a prosthetic device. This study suggests that an SMF could be used for tissue engineering for regenerating the maxillary bone.

\section{Conclusion}

Moderate-intensity (250 mT) SMF exposure increased mineralization in mouse osteoblastic MC3T3-E1 cells. However, gene expression related to decreased cell proliferation and unaltered cell differentiation and bone matrix did not correlate with the long-term effects observed following SMF exposure. The mechanism by which SMF exposure influences osteoblast mineralization remains unknown.

This work was supported by the "AGU HighTech Research Center Project" for Private Universities: matching fund subsidy from MEXT (Ministry of Education, Culture, Sports, Science and Technology), 2003-2007.

\section{References}

1. Moghadam BK, Scandrell FR. Magnetic retention overdentures. J Prosthet Dent 41: 26-29, 1979.

2. Gillings BRJ. Magnetic retention for complete and partial overdentures: Part I. J Prosthet Dent 45: 484-491, 1981.

3. Hoshiai K, Tanaka Y, Hasegawa N et al. Longitudinal study on metal plate denture with magnetic attachments: Part 4. J J Mag Dent 13(2): 26-29 2004.

4. Tanaka Y., Hiranuma K., Iwama Y et al. Sealed dental magnetic attachment developed by threedimensional magnetic analysis. Proc $10^{\text {th }}$ Int Work Shop on Rare Earth Magnet and their Applications 147-155, 1989.

5. Yan QC, Tomita N, Ikada Y. Effects of static magnetic field on bone formation of rat femurs. Med Engineer Phys 20: 397-402, 1998.

6. Yamamoto Y, Ohsaki T, Goto T et al. Effects of static magnetic fields on bone formation in rat os $^{-}$ teoblast cultures. J Dent Res 82: 962-966, 2003.

7. Camilleri S, McDonald F. Static magnetic field effects on the sagittal suture in rattus norvegicus. Am J Orthod Dentofac Orthop 103: 240-246, 1993.

8. Tsuji K, Ito Y, Noda M. Expression of the PEB-
P2alphaA/AML3/CBFA1 gene is regulated by BMP4/7 heterodimer and its overexpression suppresses type I collagen and osteocalcin gene expression in osteoblastic and nonosteoblastic mesenchymal cells. Bone 22: 87-92, 1998.

9. Zaidi SK, Javed A, Choi J et al. A specific targeting signal directs Runx2/Cbfa1 to subnuclear domains and contributes to transactivation of the osteocalcin gene. J Cell Sci 114: 3093-3102, 2001.

10. Lian JB, Roufosse AH, Reit B et al. Concentrations of osteocalcin and phosphoprotein as a function of mineral content and age in cortical bone. Calcif Tissue Int 34: S82-S87, 1982.

11. Hauschka PV, Frenkel J, DeMuth R et al. Presence of osteocalcin and related higher molecular weight 4-carboxyglutamic acid-containing proteins in developing bone. J Biol Chem. 10; 258(1): 176-182, 1983.

12. Chomczynski P, Sacchi N. Single-step method of RNA isolation by acid guanidinium thiocynatephenol-chloroform extraction. Anal Biochem162: 156-159, 1987.

13. Halpern MH and Greene AE. Effects of magnetic fields on growth of HeLa cells in tissue culture. Nature 202: 717, 1964.

14. Butler BC, Dean WW. The inhibitory effect of a magnetic field upon the tissue culture growth of the K.B. cells. Am J Med Electron 3: 123-125, 1964.

15. Feinendegen LE, Muhlensiepen H. Magnetic field affects thymidine kinase in vitro. Int $\mathrm{J}$ Rad Biol 47: 723-730, 1985.

16. Walleczek J, Liburdy RP. Nonthermal $60 \mathrm{~Hz}$ sinusoidal magnetic-field exposure enhances $45 \mathrm{Ca}^{2+}$ uptake in rat thymocytes: dependence on mitogen activation. FEBS Lett 271: 157-160, 1990.

17. Teodori L, Albertini MC, Uguccioni F et al. Static magnetic fields affect cell size, shape, orientation, and membrane surface of human glioblastoma cells, as demonstrated by electron, optic, and atomic force microscopy. Cytometry A 69: 75-85, 2005.

18. Rosen MS, Rosen AD. Magnetic field influence on paramecium motility. Life Sci 46: 1509-1515, 1990.

19. Teodori L, Gohde W, Valente MG et al. Static magnetic fields affect calcium fluxes and inhibit stressinduced apoptosis in human glioblastoma cells. Cytometry 49: 143-149, 2002.

20. Whyte MP, Vrabel LA. Infantile hypophosphatasia fibroblasts proliferate normally in culture: evidence against a role for alkaline phosphatase (tissue nonspecific isoenzyme) in the regulation of cell growth and differentiation. Calcif Tissue Int 40: $1-7,1987$.

21. Okano H, Ohkubo C. Effects of static magnetic fields on plasma levels of angiotensin II and aldosterone associated with arterial blood pressure in genetically hypertensive rats. Bioelectromagnetics 24: 403-412, 2003. 